\title{
Indicadores de saúde e sistemas de informação em saúde instrumentos para analisar a saúde da população
}

\author{
Gloria Regina da Silva e Sá \\ Omara Machado Araújo de Oliveira \\ Paula de Castro Nunes
}

\section{SciELO Books / SciELO Livros / SciELO Libros}

SÁ, G.R.S., OLIVEIRA, O.M.A., and NUNES, P.C. Indicadores de saúde e sistemas de informação em saúde: instrumentos para analisar a saúde da população. In: SILVA, M.N., FLAUZINO, R.F., GONDIM, G.M.M., eds. Rede de frio: fundamentos para a compreensão do trabalho [online]. Rio de Janeiro: Editora FIOCRUZ, 2017, pp. 133-156. ISBN: 978-65-5708-0917. https://doi.org/10.7476/9786557080917.0007.

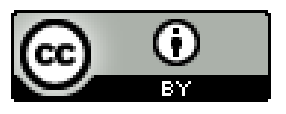

All the contents of this work, except where otherwise noted, is licensed under a Creative Commons Attribution 4.0 International license.

Todo o conteúdo deste trabalho, exceto quando houver ressalva, é publicado sob a licença Creative Commons Atribição 4.0. 


\section{Indicadores de saúde e sistemas de informação em saúde: instrumentos para analisar a saúde da população}

Gloria Regina da Silva e Sá, Omara Machado Araújo de Oliveira e Paula de Castro Nunes

A epidemiologia descritiva nos possibilitou conhecer as perguntas relacionadas a determinadas características da população, formando um conjunto de dados importantes que contribui para a análise da situação de saúde da população.

Obter dados e apresentá-los de forma organizada é muito importante. Contudo, se quisermos comparar os dados de uma doença ou um agravo que ocorre em determinado local com os de outra localidade, necessitamos de um pouco mais de conhecimento. Esses dados precisam ser transformados em informação que possa ser compreendida e analisada, comparada entre diferentes localidades e intervalos de tempo. Para tanto, a saúde pública utiliza uma série de medidas, os indicadores de saúde, traçando relações entre perguntas relacionadas a "quem", "onde" e "quando", discutidas no capítulo anterior.

Neste capítulo vamos conhecer o que são medidas em saúde pública, os indicadores de saúde, para que servem e como utilizá-los. Aqui serão apresentados elementos para que você, profissional da saúde, possa se apropriar e, consequentemente, ampliar e enriquecer o seu conhecimento na construção de uma prática mais reflexiva. Dessa forma, poderá estabelecer as necessárias relações do seu processo de trabalho com a situação de saúde e as características sociais, epidemiológicas e sanitárias da população e do seu território.

Além disso, apresentaremos, brevemente, o que são sistemas de informação em saúde e alguns exemplos, pois por meio desses sistemas são coletados dados que podem, a partir de análises, basear a criação de medidas de saúde pública/indicadores de saúde. 
Não podemos deixar de ressaltar que a saúde de uma população é avaliada, também, por indicadores indiretos, como escolaridade, renda, condições de habitação, saneamento e outros, fornecidos por setores fora da área da saúde.

\section{Medidas de saúde coletiva: quantificando a saúde}

Na epidemiologia, a quantificação dos eventos é imprescindível para se avaliar e gerenciar os serviços de saúde ofertados à comunidade e analisar a situação do processo saúde e doença de uma população em determinado território.

Essa quantificação é expressa em indicadores, ou seja, em medidas que instrumentalizam o observador para a descrição e a análise de uma situação existente, a avaliação do cumprimento de objetivos e metas e de suas mudanças ao longo do tempo, além de possibilitarem a previsão de tendências futuras.

Na epidemiologia, uma medida bastante utilizada é a frequência, que tem como principal finalidade descrever quantitativamente a ocorrência de uma doença ou um agravo à saúde. Podemos considerar dois tipos de frequência: a absoluta e a relativa.

A frequência absoluta é o resultado direto da contagem de determinada ocorrência. Esse resultado é um número bruto muito importante para o planejamento e para a gestão dos serviços de saúde, porque permite identificar o grupo da população mais atingido por determinado evento, ajudando a identificar necessidades.

\section{Veja um exemplo}

Como profissional da rede de frio, você já deve ter percebido que o quantitativo disponibilizado de determinada vacina pode oscilar.

O conhecimento do número de casos de meningite, por exemplo, permite a programação dos recursos necessários para disponibilizar a quantidade de vacinas e insumos em geral (bem como para o atendimento dos casos em relação ao número de leitos, medicamentos etc.). Essa programação será completamente diferente se tivermos uma expectativa de 20 ou 2.000 casos da doença, concorda?

A frequência relativa, por sua vez, expressa uma relação entre dois grupos de indivíduos. É uma medida que expressa uma quantidade específica, uma fração, em relação à outra quantidade. Pode ser entre:

* um grupo composto por um número parcial e outro, pelo total (por exemplo, o número de casos que evoluíram para óbito em relação ao total de casos; o número de casos de uma faixa etária em relação ao total de casos; o número de casos em relação à população); 
* dois grupos de diferentes categorias (por exemplo, o número de casos do sexo masculino em relação ao número de casos do sexo feminino).

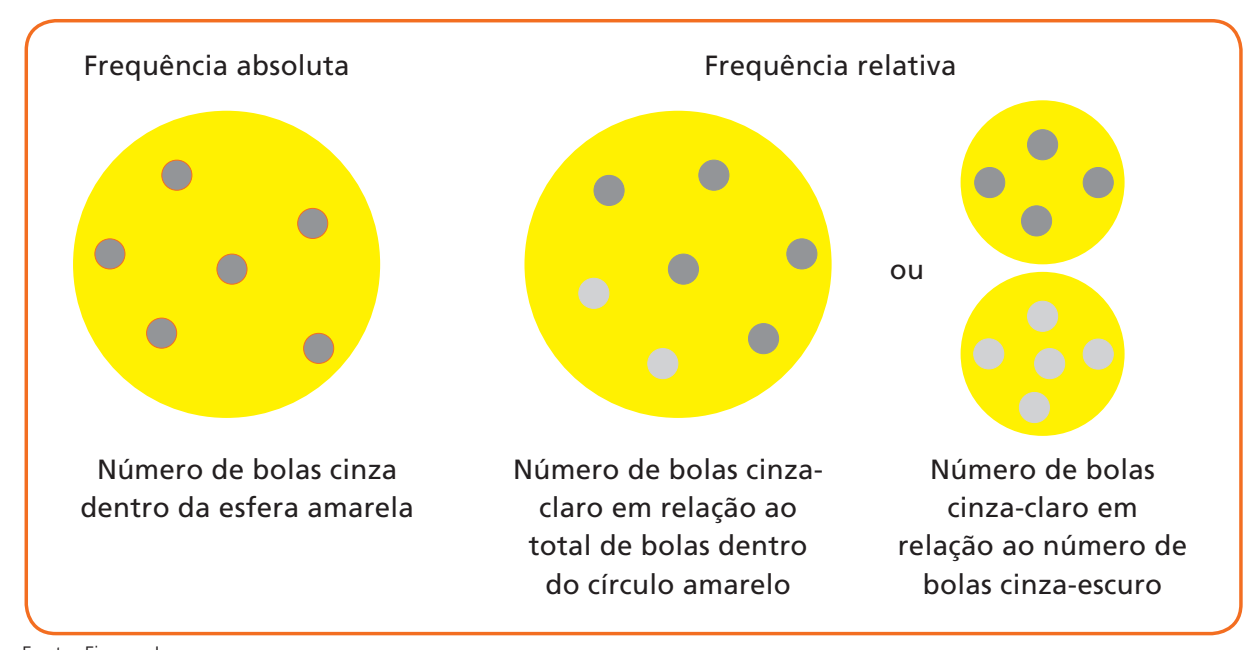

Fonte: Fiocruz Imagens.

A frequência relativa pode ser expressa em proporção, razão, coeficiente ou taxa. Em geral, utilizamos a proporção para quantificar a relação entre um número parcial e um número total de casos, enquanto o coeficiente ou taxa é utilizado para quantificar a relação entre o número de casos e a população. Por fim, a razão é utilizada para quantificar a relação entre número de casos de diferentes categorias.

$$
\begin{aligned}
& \text { Proporção }=\frac{n^{\circ} \text { parcial de casos }}{n^{\circ} \text { total de casos }} \\
& \text { Exemplo: } \frac{n^{\circ} \text { de óbitos por doenças cardiovasculares }}{n^{\circ} \text { total de óbitos }}
\end{aligned}
$$

$$
\begin{aligned}
& \begin{array}{l}
\text { Coeficiente } \\
\text { ou taxa }
\end{array}=\frac{n^{\circ} \text { de casos }}{\text { total da população }} \\
& \text { Exemplo: } \frac{n^{\circ} \text { de casos de meningite no município do Rio de Janeiro }}{\text { População do município do Rio de Janeiro }}
\end{aligned}
$$

$$
\begin{aligned}
& \text { Razão }=\frac{n^{\circ} \text { de casos do tipo } X}{n^{\circ} \text { de casos do tipo } Z} \\
& \text { Exemplo: } \frac{n^{\circ} \text { de casos de Aids no sexo masculino }}{n^{\circ} \text { de casos de Aids no sexo feminino }}
\end{aligned}
$$




\section{Indicadores de saúde}

A despeito de termos indicado as frequências como medidas da saúde coletiva, a partir de agora abordaremos mais especificamente os indicadores de saúde mais utilizados para avaliar a situação de saúde de uma população e orientar o desenvolvimento de políticas públicas.

\section{Rede Interagencial de Informações para a Saúde (Ripsa)}

Formalizada em 1996, por portaria ministerial e por acordo de cooperação

Na Ripsa, você pode aprofundar os indicadores de saúde que apresentamos a seguir e diversos outros. Para isso, acesse a http://www.ripsa. org.br/vhl/indicadores-e-dadosbasicos-para-a-saude-no-brasilidb/ficha-de-qualificacao-doindicador/ com a OPAS, a Ripsa tem como propósito promover a disponibilidade adequada e oportuna de dados básicos, indicadores e análises sobre as condições de saúde e suas tendências, visando aperfeiçoar a capacidade de formulação, gestão e avaliação de políticas e ações públicas pertinentes. O principal instrumento de orientação técnica da Ripsa são as Fichas de Qualificação, que expõem os conceitos e critérios específicos para a construção de um indicador. As Fichas de Qualificação podem ser acessadas no site: http://fichas.ripsa.org.br/2012/.

Fonte: Rede Interagencial de Informações para a Saúde (2012)

Os indicadores mais utilizados em saúde para avaliar, em populações diferentes, os riscos de adoecer ou morrer são, respectivamente, os de morbidade e mortalidade. Mortalidade e morbidade são medidas relativas e sempre se referem a uma população específica e a um intervalo de tempo determinado.

\section{Principais indicadores de morbidade}

A morbidade expressa o comportamento de uma doença ou de um agravo à saúde em uma população exposta, em determinado período. É medida pelos coeficientes ou taxas de incidência e prevalência.

\section{Coeficiente ou taxa de incidência}

A incidência demonstra a intensidade com que uma doença ou um agravo ocorre na população. É a intensidade da morbidade. É calculada pelo coeficiente de incidência, que mede a ocorrência de casos novos da doença ou outros eventos na população.

Esse coeficiente é definido como a relação entre o número de casos novos de determinada doença ou agravo e a população exposta ao risco de adquirir a doença ou sofrer determinado agravo à saúde. Portanto, é uma medida de risco. É muito utilizada quando são estudados os fatores causais de adoecimento, porque pode ser calculada por sexo, grupo etário, regiões, situação de exposição. 
A unidade de tempo (período em análise) pode ser dia, mês, semana, ano, dependendo da doença em estudo ou do interesse em se obter medidas ao longo do tempo. A unidade de referência é "habitantes" (ou "habitantes por especificação", dependendo da população exposta na análise; por exemplo, alunos, quando se referir à incidência de casos em uma escola).

\section{Justificativa da potência de 10 nas fórmulas}

Para facilitar e permitir a comparação entre os coeficientes, tanto os de mortalidade quanto os de morbidade, calculados para diferentes locais ou para o mesmo local, em diferentes períodos de tempo, o resultado utiliza sempre uma base comum $(100,1.000,10.000,100.000$, 1.000.000), que representa uma potência de $10\left(10^{n}\right)$. Essa potência de 10 é escolhida de forma a tornar os números obtidos o mais próximo possível do inteiro, procurando aumentar as frações obtidas pela divisão e, consequentemente, diminuindo o número de zeros dessas frações decimais. Por convenção, nos coeficientes de mortalidade geral e infantil, a base é por 1.000 , e, quando se trata de mortalidade por causas, a base mais adequada é $10^{5}=100.000$. Expressa-se o coeficiente de letalidade sempre em porcentagem.

A seguir apresentamos a fórmula para o cálculo do coeficiente de incidência e de prevalência.

\section{Fórmula para o cálculo do coeficiente de incidência $(\mathrm{Cl})$ \\ $\mathrm{Cl}=\frac{\mathrm{n}^{\circ} \text { de casos novos de uma doença, num dado local e período } \times 10^{n}}{\text { População do mesmo local e período }}$}

Exemplo: coeficiente de incidência de tuberculose, Salvador, 2014

- 7.805 casos novos em 2014

- população do estado em 2014: 12.709.744 habitantes

Coeficiente de incidência $=\frac{7.805}{12.709 .744} \times 10.000=6,14 / 10.000$ hab.

Interpretando: ocorreram, aproximadamente, seis casos novos de tuberculose em cada grupo de 10 mil habitantes, em Salvador, no ano de 2014.

Essa informação pode dar ao profissional de saúde a dimensão da magnitude da doença, ou seja, a força do risco de adoecer de tuberculose na população. Com esse conhecimento da incidência, é possível, para o profissional, planejar as ações de forma preventiva, bem como intervir de modo a evitar a propagação ou disseminação da doença por meio de medidas, como a vacinação. 


\section{Coeficiente ou taxa de prevalência}

Prevalência é o número total de casos existentes em determinado local e período. É calculado pelo coeficiente de prevalência, que mede a frequência ou a probabilidade de existência de casos (novos e antigos) da doença na população. Permite estimar e comparar, no tempo, a persistência de determinada doença, sendo mais utilizado para análise de doenças crônicas. É um coeficiente importante no planejamento da assistência à saúde, porque possibilita, por exemplo, calcular a quantidade de medicamentos ou leitos hospitalares necessária. Da mesma forma que no coeficiente de incidência, os resultados podem ser multiplicados por uma base referencial, que é uma potência de $10\left(10^{\mathrm{n}}\right)$.

\section{Fórmula para o cálculo do coeficiente de prevalência (CP)}

$n^{\circ}$ de total de casos (novos e antigos)

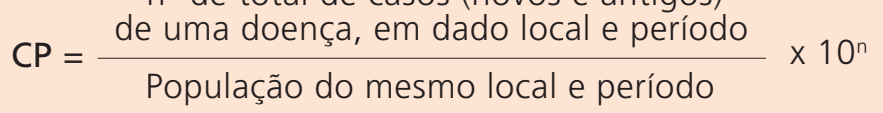

Exemplo: coeficiente de prevalência de tuberculose, Salvador, 2014

- 7.805 casos novos foram diagnosticados naquele ano

- 2 mil tiveram cura por alta

- 30 foram a óbito

- 400 novos diagnosticados em 2009 persistiam doentes por causa de resistência ao tratamento

Numerador $=7.805+400-2.000-30=6.175$

Coeficiente de prevalência $=\frac{6 \cdot 175}{12 \cdot 709.744} \times 10.000=4,86 / 10.000$ hab.

Interpretando: existiam, aproximadamente, em 2014, cinco pacientes em tratamento de tuberculose em cada 10 mil habitantes.

Essa informação fornece ao profissional de saúde a dimensão da força com que a doença subsiste na população, possibilitando o planejamento das ações e dos recursos necessários ao tratamento, como aquisição de medicamentos, previsão do número de leitos e de profissionais necessários ao atendimento desse agravo.

\section{Taxa ou coeficiente de ataque}

Está padronizado que o seja expresso em porcentagem Ou seja, a potência de 10 utilizada é $10^{2}=100$.
É o coeficiente de incidência (ou seja, número de casos novos em determinados período e local) em uma população específica exposta ao risco; limitado a uma área e tempo restritos. 
Fórmula para o cálculo da taxa de ataque (TA)

$\mathrm{TA}=\frac{\mathrm{n}^{\circ} \text { de casos novos de uma doença, num dado local e período }}{\text { População exposta ao risco }} \times 100$

A taxa de ataque é muito útil para identificar a fonte de infecção, ao se investigarem ou se analisarem surtos epidêmicos de determinada doença, em um local ou em uma situação em que o grupo populacional é bem definido, como residência, creche, escola, quartel, ou quando se trata de um grupo de pessoas que participou de um evento específico, como um almoço ou um lanche. Por exemplo, em uma escola que possui 100 alunos, 20 apresentaram uma infecção intestinal. A TA neste caso seria:

$$
\begin{aligned}
& \mathrm{TA}=\frac{20}{100} \times 100 \\
& \mathrm{TA}=20 \%
\end{aligned}
$$

\section{Ressaltando}

Os indicadores de morbidade que tratam do risco de adoecer são o coeficiente de incidência e o coeficiente ou taxa de ataque. O risco de morrer pode ser expresso por diversos indicadores, como o coeficiente geral e específico de mortalidade e o coeficiente ou taxa de letalidade, e cada um deles é apresentado a seguir.

\section{Principais indicadores de mortalidade}

A mortalidade é uma variável característica das comunidades de seres vivos e refere-se ao conjunto dos indivíduos que morreram em dado intervalo do tempo. Os coeficientes de mortalidade são definidos como quocientes entre as frequências absolutas de óbitos e o número dos expostos ao risco de morrer.

\section{Coeficiente de mortalidade geral}

O coeficiente de mortalidade é a relação entre a frequência absoluta, ou seja, a soma ou total dos óbitos e o número total de pessoas expostas ao risco de morrer. O coeficiente de mortalidade é geral quando inclui todos os óbitos e toda a população da área em estudo.

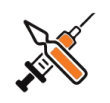

Por convenção, para o cálculo da taxa de mortalidade geral e infantil, a potência de 10, padronizada internacionalmente, é $10^{3}$ (1.000). 
Fórmula para o cálculo do coeficiente de mortalidade geral (CMG)

$\mathrm{CMG}=\frac{\mathrm{n}^{\circ} \text { total de óbitos em determinada área e período }}{\text { População da mesma área e no mesmo período }} \times 1.000$

Exemplo: coeficiente de mortalidade geral do município do Rio de Janeiro, 2012

- Total de óbitos em 2012: 52.263

- População do município em 2012: 6.390.290 habitantes

Coeficiente de mortalidade geral $=\frac{52.263}{6.390 .290} \times 1.000=8,18 / 1.000$ hab.

Interpretando: em 2012, no município do Rio de Janeiro, morreram oito pessoas para cada mil habitantes.

Apesar de ser utilizado na saúde pública, o seu uso torna-se limitado pela qualidade de registro de óbitos e pela dificuldade de comparação internacional devida às diferenças nas estruturas etárias. Por exemplo, em países desenvolvidos, a estrutura etária da população é composta de um grande percentual de idosos, o que aumenta o CMG e pode fornecer uma falsa impressão de que o risco de morrer seja maior nesses do que nos países menos desenvolvidos. Por esse motivo, quando se deseja comparar a mortalidade de duas áreas de composições etárias diferentes, faz-se necessária a padronização das taxas.

A tabela, a seguir, apresenta o coeficiente de mortalidade geral padronizado no Brasil, por regiões e sexo. Observamos que, em 2008, o coeficiente de mortalidade geral foi de 4,92 óbitos por mil habitantes. Em todas as regiões, esse coeficiente possui maior valor no sexo masculino.

Tabela 1 - Número e proporção de óbitos e coeficiente de mortalidade geral padronizado por mil hab - Brasil, 2008

\begin{tabular}{|c|c|c|c|c|c|c|c|c|}
\hline \multirow[t]{2}{*}{ Regiões } & \multirow[t]{2}{*}{$\begin{array}{l}\text { Número de } \\
\text { óbitos }\end{array}$} & \multirow[t]{2}{*}{$\begin{array}{l}\% \text { de } \\
\text { óbitos }\end{array}$} & \multicolumn{3}{|c|}{$\begin{array}{l}\text { Coeficiente de mortalidade geral } \\
\text { bruto (por mil habitantes) }\end{array}$} & \multicolumn{3}{|c|}{$\begin{array}{l}\text { Coeficiente de mortalidade geral } \\
\text { padronizado (por mil habitantes) }\end{array}$} \\
\hline & & & Total & Homens & Mulheres & Total & Homens & Mulheres \\
\hline Nordeste & 269.870 & 25,30 & 5,07 & 5,97 & 4,22 & 4,70 & 5,54 & 3,89 \\
\hline Centro-Oeste & 66.545 & 6,24 & 4,86 & 5,98 & 3,75 & 4,86 & 5,75 & 4,00 \\
\hline Brasil & 1.066 .842 & 100 & 5,62 & 6,58 & 4,70 & 4,92 & 5,82 & 4,05 \\
\hline
\end{tabular}

Fonte: Brasil (2010). 


\section{Coeficiente de mortalidade por causa}

Representa o número de óbitos por uma causa específica em relação ao total da população. Por exemplo, número de mortos por tuberculose em relação ao número total de mortos na população. Muito usado, fornece dados importantes sobre a saúde das coletividades. Quando acompanha as doenças transmissíveis, mede, indiretamente, as condições de saneamento e a eficiência dos serviços de prevenção e programas de controle de doenças.

\section{Fórmula para o cálculo do coeficiente de mortalidade} específica por causa (CMEC)

CMEC $=\frac{n^{\circ} \text { de óbitos por determinada causa em um dado período }}{\text { População }} \times 100.000$

Exemplo: coeficiente de mortalidade específica por causa externa no estado de São Paulo, 2012

- Óbitos por causas externas em 2012: 25.041

- População do estado em 2012: 41.901.219

Coeficiente de mortalidade por causas externas

$$
=\frac{25.041}{41.901 .219} \times 100.000=59,8 / 100.000 \text { hab. }
$$

Interpretando: em São Paulo, ocorreram aproximadamente 60 óbitos por causas externas para cada 100 mil habitantes no ano de 2012.

Na tabela a seguir, são apresentados os coeficientes de mortalidade específicos para as dez principais causas de óbito definidas (por 100 mil habitantes) segundo sexo, no Brasil, no ano de 2008. Observamos que, em todo país, entre as mulheres, a primeira causa de óbito são as doenças cerebrovasculares, entre os homens são as doenças isquêmicas do coração, com participação importante das agressões.

Tabela 2 - Número absoluto de óbitos, proporção (\%) e coeficientes de mortalidade brutos específicos (por 100 mil habitantes) para as dez principais causas de óbito definidas, total e segundo sexo - Brasil, 2008

\begin{tabular}{|c|c|c|c|c|c|c|c|c|c|c|}
\hline \multirow[t]{2}{*}{ Ordem } & \multirow[t]{2}{*}{ Causas } & \multicolumn{3}{|l|}{ Total } & \multicolumn{3}{|l|}{ Homens } & \multicolumn{3}{|l|}{ Mulheres } \\
\hline & & $\mathrm{N}^{\circ}$ & $\%$ & CMB & $\mathrm{N}^{\circ}$ & $\%$ & CMB & $\mathrm{N}^{\circ}$ & $\%$ & CMB \\
\hline 1 & $\begin{array}{l}\text { Doenças } \\
\text { cerebrovasculares }\end{array}$ & 97.881 & 9,2 & 51,6 & 49.311 & 8,0 & 52,9 & 48.563 & 10,7 & 50,3 \\
\hline 2 & $\begin{array}{l}\text { Doenças isquêmicas } \\
\text { do coração }\end{array}$ & 94.912 & 9,9 & 50,1 & 55.162 & 9,0 & 59,2 & 39.744 & 8,8 & 41,2 \\
\hline 4 & Diabetes mellitus & 49.683 & 4,7 & 26,2 & 21.639 & 3,5 & 23,2 & 28.040 & 6,2 & 29,1 \\
\hline
\end{tabular}


Tabela 2 - Número absoluto de óbitos, proporção (\%) e coeficientes de mortalidade brutos específicos (por 100 mil habitantes) para as dez principais causas de óbito definidas, total e segundo sexo - Brasil, 2008 (cont.)

\begin{tabular}{|c|c|c|c|c|c|c|c|c|c|c|}
\hline \multirow[t]{2}{*}{ Ordem } & \multirow[t]{2}{*}{ Causas } & \multicolumn{3}{|l|}{ Total } & \multicolumn{3}{|l|}{ Homens } & \multicolumn{3}{|l|}{ Mulheres } \\
\hline & & $\mathrm{N}^{\circ}$ & $\%$ & CMB & $\mathrm{N}^{\circ}$ & $\%$ & CMB & $\mathrm{N}^{\circ}$ & $\%$ & CMB \\
\hline 5 & Agressões & 48.610 & 4,6 & 25,6 & 44.671 & 7,3 & 48,0 & 3.880 & 0,9 & 4,0 \\
\hline 7 & Doenças hipertensivas & 42.167 & 4,0 & 22,2 & 19.909 & 3,2 & 21,4 & 22.254 & 4,9 & 23,1 \\
\hline 8 & Acidentes de transporte & 37.585 & 3,5 & 19,8 & 30.668 & 5,0 & 32,9 & 6.894 & 1,5 & 7,1 \\
\hline \multirow[t]{2}{*}{10} & $\begin{array}{l}\text { Restante de } \\
\text { neoplasias malignas }\end{array}$ & 30.458 & 2,9 & 16,1 & 15.437 & 2,5 & 16,6 & 15.017 & 3,3 & 15,6 \\
\hline & Todas as causas & 1.066 .842 & 100,0 & 562,6 & 612.858 & 100,0 & 657,9 & 453.524 & 100,0 & 470,2 \\
\hline
\end{tabular}

\section{Coeficiente de mortalidade infantil}

É a relação entre os óbitos em crianças com menos de um ano de idade e o número de nascidos vivos, em determinada área e período de tempo.

Fórmula para o cálculo do coeficiente de mortalidade infantil (CMI)

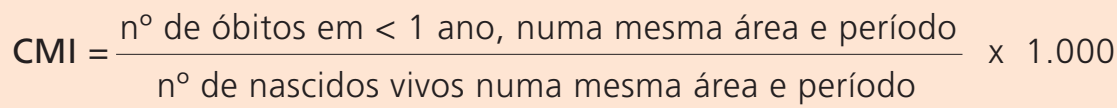

Exemplo: coeficiente de Mortalidade Infantil no estado do Amazonas, 2013.

- Total de óbitos de < 1 ano no AM em 2013: 1.361

- Total de nascidos vivos no estado em 2013: 79.041

$\begin{aligned} & \text { Coeficiente de mortalidade } \\ & \text { infantil }\end{aligned}=\frac{1.361}{79.041} \times 1.000=17,22 / 1.000 \begin{aligned} & \text { nascidos } \\ & \text { vivos }\end{aligned}$

Interpretando: no estado do Amazonas, em 2013, morreram aproximadamente 17 crianças antes de completarem 1 ano de vida para cada mil crianças nascidas vivas.

O coeficiente de mortalidade infantil é uma estimativa do risco de morte a que está exposta uma população de nascidos vivos, em determinada área e período, antes de completar o primeiro ano de vida. É um indicador muito utilizado em saúde pública, por refletir as condições de vida de uma população, uma vez que a criança, nessa faixa de idade, é extremamente sensível a todas as condições socioeconômicas e ambientais. Utiliza-se o número de nascidos vivos como denominador, pois ele produz uma estimativa mais acurada do que a população com menos de 1 ano de idade. 
O gráfico a seguir demonstra a evolução do coeficiente de mortalidade infantil no Brasil, no período de 1990 a 2008, período no qual observamos uma queda bastante acentuada desse indicador. O seu valor passou de 53,7 para 24,1 óbitos por mil nascidos vivos. Contudo, persistem diferenças inter-regionais importantes. Desde a década de 1990, as regiões com as maiores e as menores taxas foram a Nordeste e a Sul, respectivamente. Em 1990, a mortalidade na Região Nordeste era 2,5 vezes maior do que na Sul.

Gráfico 1 - Evolução do coeficiente de mortalidade infantil (por mil nascidos vivos). Brasil e regiões, 1990 a 2008* e projeção até 2015

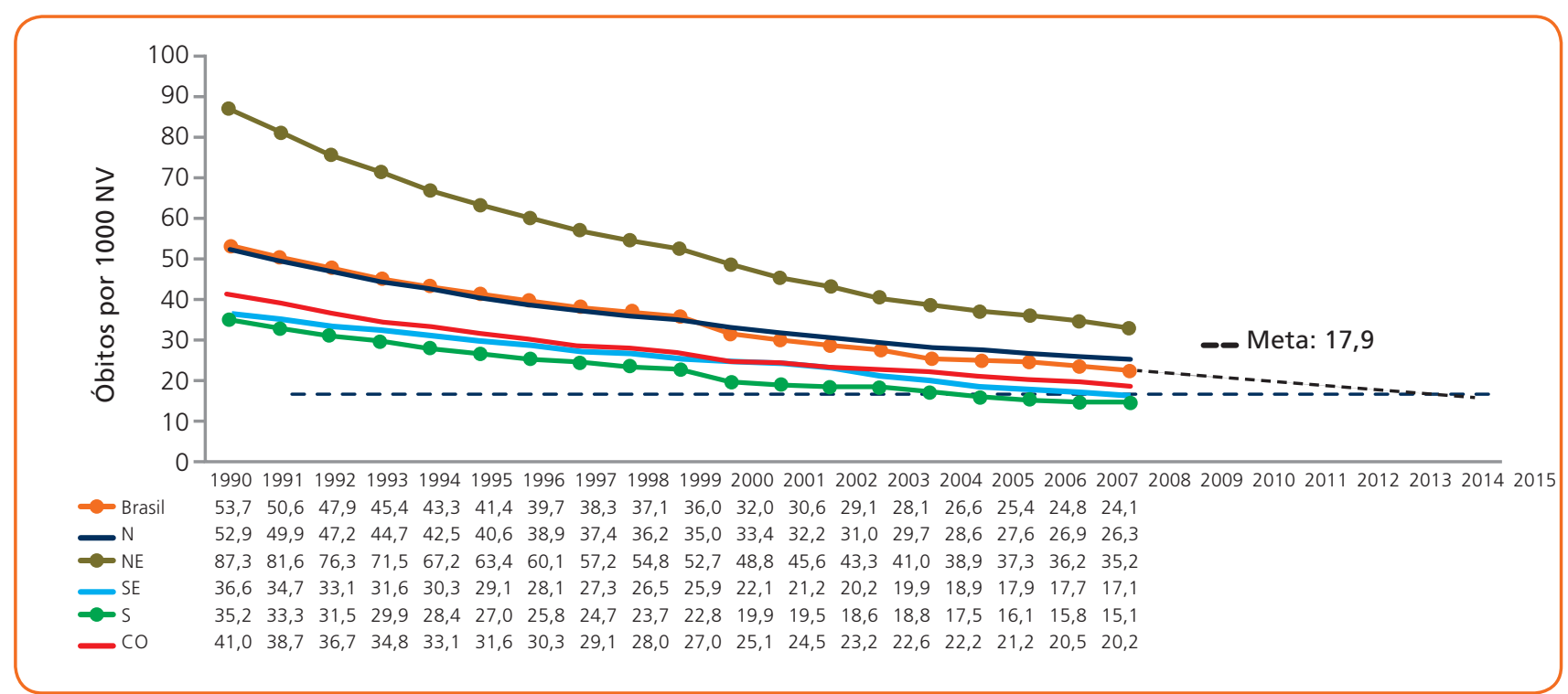

* Dados preliminares.

Fonte: Brasil (2010).

\section{Coeficiente de mortalidade materna}

É o quociente entre o número de mortes por causas maternas e o número de nascidos vivos em determinado período e área. Mede o risco de morte de mulheres por causas maternas (causas relacionadas à gravidez, ao parto e ao puerpério). 
Morte materna, segundo a $10^{\text {a }}$ Revisão da Classificação Internacional de Doenças (CID10), é a "morte de uma mulher durante a gestação ou até 42 dias após o término da gestação, independente da duração ou da localização da gravidez, devida a qualquer causa relacionada com ou agravada pela gravidez ou por medidas em relação a ela, porém não devida a causas acidentais ou incidentais" (CID-10, 2008).

\section{Fórmula para o cálculo do coeficiente de mortalidade materna (CMM)}

$\mathrm{n}^{\circ}$ de óbitos por causas maternas

$\mathrm{CMM}=\frac{\text { em determinados local e período }}{\text { Total de nascidos vivos }} \times 100.000$

Exemplo: coeficiente de mortalidade materna no Brasil, 2013

- Total de óbitos maternos em 2013: 1.686

- Total de nascidos vivos no Brasil em 2013: 2.904.027

$\begin{aligned} & \text { Coeficiente de } \\ & \text { mortalidade } \\ & \text { materna }\end{aligned}=\frac{1.686}{2.904 .027} \times 100.000=58,05 / 100.000$ nascidos vivos

Interpretando: no Brasil, em 2013, ocorreram aproximadamente 58 óbitos maternos para cada 100 mil nascidos vivos.

O CMM é um instrumento de análise das condições de assistência pré-natal e obstétrica. O CMM observa o impacto negativo da assistência, deficiente e/ou de má qualidade, para a população de gestantes, parturientes e puérperas. A base utilizada, por convenção, é de 100 mil nascidos vivos.

O denominador ideal para o CMM é a população de mulheres gestantes, pois são estas que estão sob risco de morrer por causas maternas. Entretanto, como é difícil obter-se uma estimativa desse número para a população geral, utiliza-se o número de nascidos vivos na área e no período de interesse. Os nascidos vivos são utilizados, partindo-se do pressuposto que a proporção de natimortos (nascidos mortos) no total de nascimentos é desprezível (ou seja, que a maioria das gestações produz nascidos vivos).

No gráfico a seguir, apresentamos o coeficiente de mortalidade materna nos anos de 1990, 2000 e 2007. Nele, observamos uma diminuição de todas as causas específicas de morte materna, o que pode sugerir melhora nas condições de assistência pré-natal e obstétrica. 
Gráfico 2 - Coeficiente de mortalidade materna por causa específica de morte (por 100 mil nascidos vivos). Brasil, 1990, 2000 e 2007

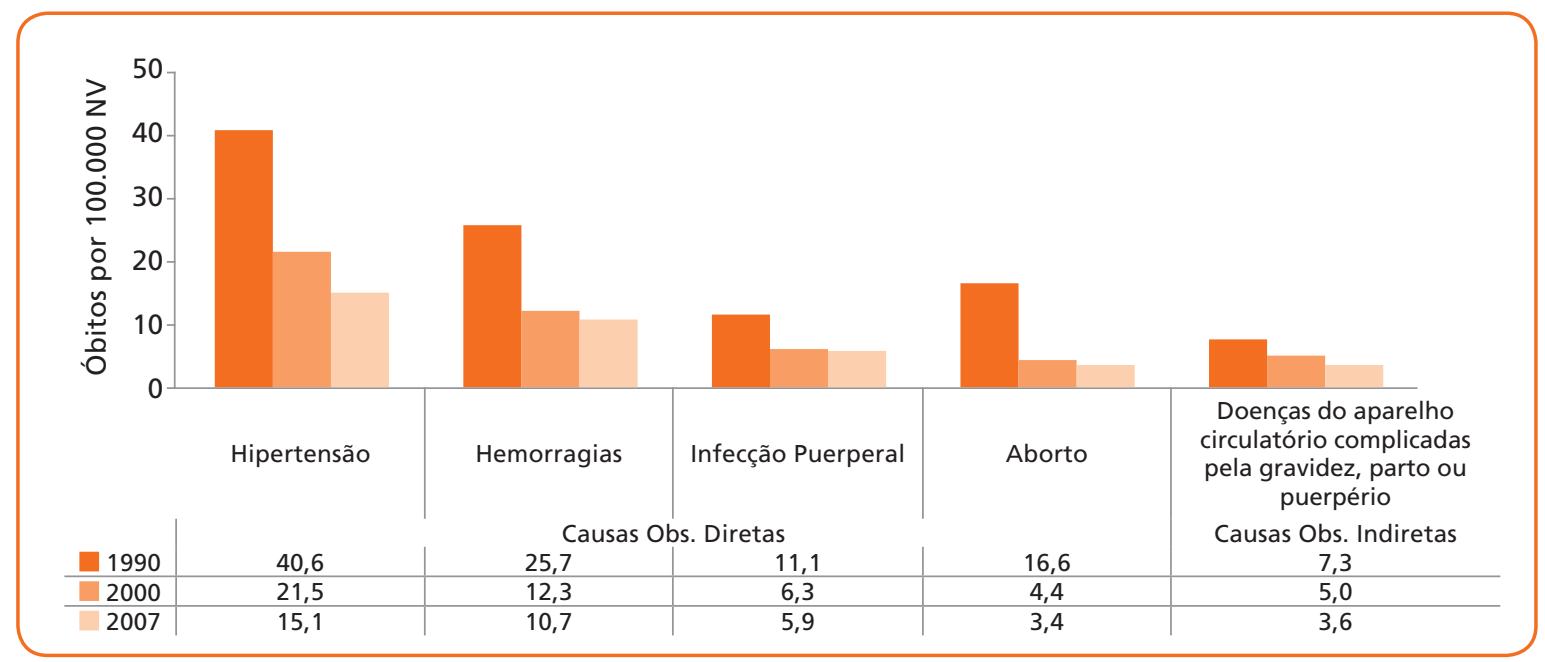

Fonte: Brasil (2010).

\section{Mortalidade proporcional}

É um indicador que permite analisar a distribuição proporcional dos óbitos em relação a algumas variáveis de interesse, como idade e causa do óbito, isto é, quando se deseja avaliar a frequência de um evento em relação ao total de óbitos.

É importante ressaltar que esse indicador não mede o risco de morrer, e sim a concentração dos óbitos por determinada variável de interesse. Pode ser utilizado quando não estão disponíveis dados de população, pois já é um indicativo da situação de mortalidade do local que está sendo avaliado.

\section{Mortalidade proporcional por determinada causa, idade} ou sexo

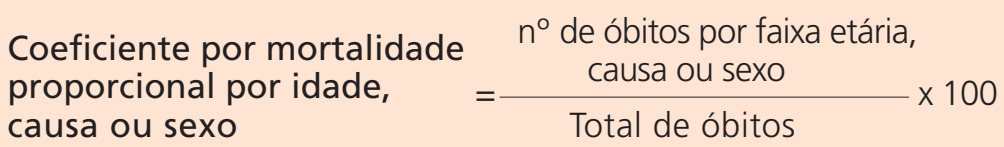

Exemplo: mortalidade proporcional de 50 e mais anos de idade, Brasil, 2013

- total de óbitos de 50 e mais anos no Brasil em 2013: 917.697

- total de óbitos no Brasil em 2013: 1.201.474 $\begin{aligned} & \text { Coeficiente de mortalidade } \\ & \text { proporcional de } 50 \text { anos ou mais }\end{aligned}=\frac{917.697}{1.201 .474} \times 100=76 \%$

Interpretando: no Brasil, em 2013, a cada cem óbitos, 76 estavam na faixa etária de 50 e mais anos de idade.

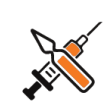

Por convenção, o resultado da mortalidade proporcional é expresso em porcentagem, a potência de 10 utilizada é $10^{2}=100$. 
O Gráfico 3 apresenta a série histórica da mortalidade proporcional no Brasil; observe que há mudança nos padrões de adoecimento, pois o acesso à saúde e a expectativa de vida influem diretamente nos valores de mortalidade proporcional.

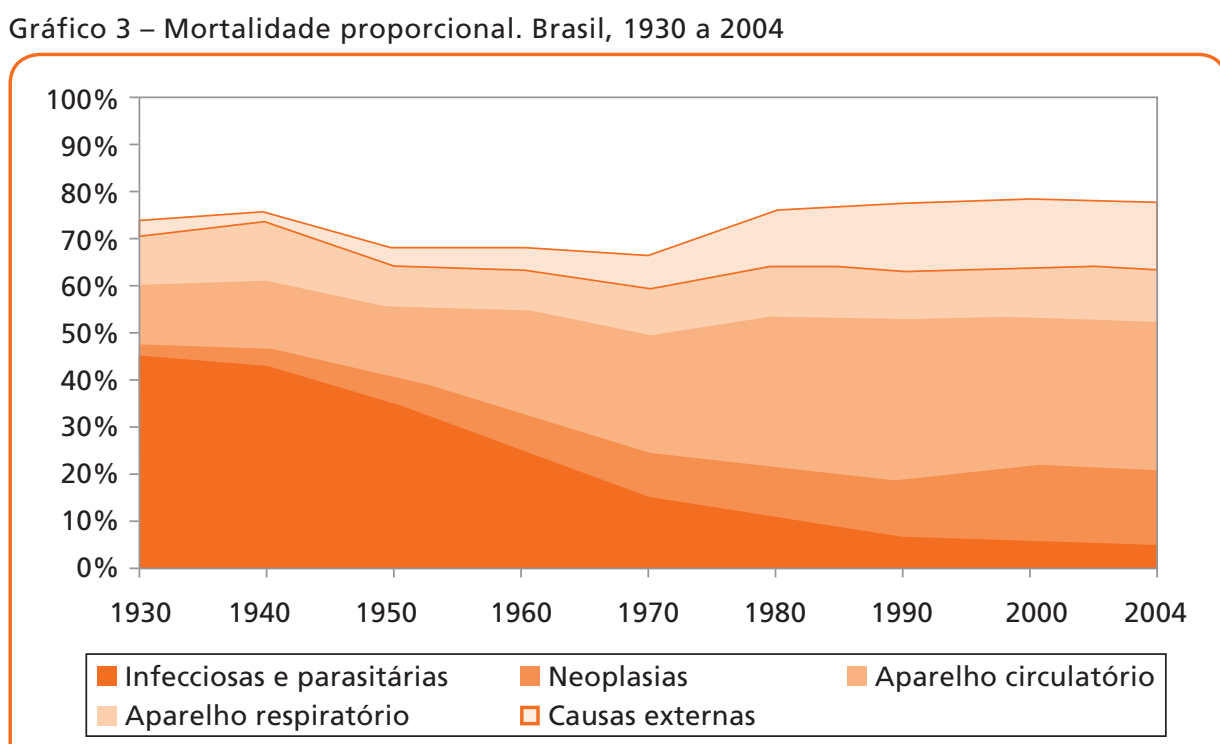

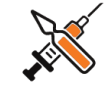

A taxa de letalidade é expressa, sempre, em porcentagem.
Fonte: Rouquayrol; Almeida (2013).

\section{Taxa de letalidade}

A taxa de letalidade (TL) pode ser definida como o maior ou menor poder que uma doença tem de provocar a morte nas pessoas. Mede o risco de morrer de determinado agravo ou doença.

A TL relaciona o número de óbitos por determinada causa com o número de pessoas acometidas por essa doença. Essa relação nos dá uma ideia da gravidade do problema, pois indica o percentual de indivíduos que morrem por tal doença.

\section{Fórmula para o cálculo da taxa de letalidade (TL)}

$\mathrm{TL}=\frac{\mathrm{n}^{\circ} \text { de óbitos por uma doença, num dado local e período }}{\text { total de casos da doença do mesmo local e período }} \times 100$

Por exemplo: em uma festa de casamento, foi oferecido um salpicão com maionese deteriorada, gerando um surto de intoxicação alimentar. Dos convidados presentes, cem consumiram o alimento e ficaram doentes; destes, dez não resistiram à intoxicação e vieram a falecer. Avaliando a taxa de letalidade, observou-se que foi de $10 \%$. 


$$
\begin{aligned}
& T L=\frac{10}{100} \times 100 \\
& T L=10 \%
\end{aligned}
$$

\section{Outros indicadores}

O acompanhamento do Programa Nacional de Imunizações (PNI) é feito pelos indicadores de cobertura vacinal (CV) por cada vacina, e existem metas de alcance de cobertura mínima para cada vacina específica. A meta de CV para a vacina tríplice viral, por exemplo, é de $\geq 95 \%$ na população de 1 ano de idade; a meta da CV mínima para a pentavalente é de $\geq 90 \%$ com a $3^{\text {a }}$ dose.

\section{Cobertura vacinal$$
\mathrm{CV}=\frac{\mathrm{n}^{\circ} \text { de doses aplicadas na população-alvo (por determinada vacina) }}{\text { população-alvo (em determinado período e lugar) }} \times 100
$$

Outro indicador do PNI é homogeneidade de CV, cujo cálculo é feito colocando-se, no numerador, o número de municípios que atingiram a cobertura de $95 \%$ ou mais e, no denominador, o número total de municípios do estado, multiplicado por 100. Esse indicador nos dá uma visão mais ampla do percentual do estado que está coberto pela vacinação e nos mostra onde podem existir bolsões de população suscetível.

$$
\begin{aligned}
& \text { Homogeneidade da cobertura vacinal (HCV) } \\
& \mathrm{HCV}=\frac{\mathrm{n}^{\circ} \text { de municípios que atingiram cobertura } \geq 95 \%}{n^{\circ} \text { total de municípios do estado }} \times 100
\end{aligned}
$$

Todos esses indicadores só podem ser construídos com base em um conjunto de dados que foi coletado, registrado para que pudesse ser analisado e transformado nos indicadores que vimos há pouco. Utilizamos, em saúde, um conjunto de sistemas de informação para esta função, que tem grande importância para o registro dos dados de saúde da população, os quais subsidiam a tomada de decisões das instâncias de gestão do SUS.
Além de cobertura vacinal e homogeneidade da cobertura, outros indicadores são apresentados no Capítulo 4, "Avaliação da vacinação", do livro Rede de Frio: gestão, especificidades e atividades (SILVA; FLAUZINO, 2016). 


\section{Sistemas de informação em saúde}

Para que possamos nos comunicar, é necessário que, em nossa mente, sejam elaboradas informações que possam ser transmitidas a outras pessoas. Mas, como isso acontece?

Sabemos que o nosso cérebro recebe estímulos de várias fontes, sejam elas visuais, auditivas, táteis, olfativas e gustativas. Ao receber esses estímulos, o cérebro se assemelha a um grande sistema. A partir do momento que armazena esses dados, os integra, processa, transforma e os libera sob a forma de informações que permitem a troca, estabelecendo a comunicação entre as pessoas.

Os dados de saúde são transformados em informações pertinentes e importantes para a comunicação e avaliação na área da saúde, semelhante ao que acontece no cerebro. A informação em saúde é um recurso fundamental para a tomada de decisões, o planejamento, a execução e a avaliação de ações. Em sua concepção, a informação é obtida por uma combinação de dados. Os dados são considerados eventos e acontecimentos como, por exemplo, nascimento, óbito, casos de doenças, internação hospitalar, gestante acompanhada no pré-natal, dose de vacina aplicada, criança vacinada etc.

Podemos perguntar, então: de que forma é possível reunir dados para obter informações? Uma forma de reunir dados para conhecer determinada situação é organizar um sistema. Sistema é a disposição de elementos, ou seja, de dados, de um todo, coordenados entre si e que funciona como uma estrutura organizada (BRASIL, 2009).

Para que um sistema de informação possa reunir, consolidar e analisar dados para produzir informações, é fundamental a obtenção de todos os dados. Para isso, devemos identificar e cadastrar todas as fontes, ter instrumentos de coleta apropriados e estabelecer um fluxograma de hierarquia entre as fontes e os componentes do sistema, de forma a reunir, consolidar os dados e produzir informações.

A fonte do dado é o local onde ocorre o evento, ou seja, onde o evento é produzido. Assim, por exemplo, se a fonte de dados de nascimentos é onde ocorre o parto, ou seja, nas maternidades, o sistema montado para coletar dados de nascimentos deve incluir, obrigatoriamente, como fonte, todas as maternidades. 
Os sistemas de informação em saúde (SIS) permitem a coleta, o armazenamento, o processamento, a recuperação e disseminação de informações sobre a saúde da população, apoiando as funções operacionais, gerenciais e de tomada de decisão de interesse da área da saúde.

Oportunidade, atualidade, disponibilidade e cobertura são características que determinam a qualidade da informação e são fundamentais para que todo SIS apresente um bom desempenho.

Dependem da concepção apresentada, da sua sensibilidade para captar, o mais precocemente possível, as alterações que podem ocorrer no perfil de morbimortalidade de uma população em sua área e, também, na organização e cobertura das atividades desenvolvidas.

Na concepção do Sistema Único de Saúde (SUS), um dos objetivos básicos dos SIS é possibilitar, por meio da construção de indicadores, a análise da situação de saúde da população, considerando, necessariamente, suas condições de vida na determinação do processo saúde-doença.

O nível local tem grande responsabilidade na alimentação do sistema de informação em saúde, como também na sua organização e gestão. Por ser considerado uma fonte de dados, é de primordial importância que os dados sejam registrados em instrumentos, de maneira adequada, de forma que as informações ali geradas possam identificar as vulnerabilidades e os riscos aos quais a população está sujeita e possam ser implantadas as intervenções necessárias para prevenção e controle destes.

Percebe-se, nessa situação, que o profissional da saúde é figura fundamental na alimentação do sistema de informação em saúde. Por exemplo, quando em uma sala de vacinação o profissional de saúde, sistematicamente, deixa de registrar dados relativos a pessoas vacinadas e vacinas administradas, este fato irá interferir tanto no processo de planejamento para cálculo de cobertura vacinal como também contribuir para o cálculo inadequado de estoque de imunobiológicos. Isso acarreta uma visão não condizente com a realidade de cobertura vacinal daquela população assistida e o risco de suspensão de vacinação, em virtude da falha na regulação do estoque.

É importante ressaltar que a informação é fundamental para a democratização da saúde e o aprimoramento da sua gestão. Por esse motivo, a informatização das atividades do SUS é essencial para a descentralização das atividades de saúde e a viabilização do controle social sobre a utilização dos recursos disponíveis. Para que fosse possível alcançar 
No Capítulo 6, "Vigilância epidemiológica", são apresentadas algumas DNC, bem como a indicação da portaria do Ministério da Saúde que determina quais doenças devem ser obrigatoriamente notificadas.

Vale ressaltar que, com a intensificação da vacinação, será necessário o reforço no quantitativo de vacinas a serem administradas; a logística da rede de frio deve estar preparada para atender a essas necessidades. esses objetivos, foi criado o Departamento de Informática do SUS, o DATASUS (2011), com a missão de:

prover os órgãos do SUS de sistemas de informação e suporte de informática, necessários ao processo de planejamento, operação e controle do Sistema Único de Saúde, por meio da manutenção de bases de dados nacionais, apoio e consultoria na implantação de sistemas e coordenação das atividades de informática inerentes ao funcionamento integrado dos mesmos (DATASUS, [201-]).

Estão integrados no DATASUS todos os sistemas que compõem o sistema de informação do SUS, o que possibilita a obtenção e o processamento de grande volume de dados, além de permitir a articulação entre diferentes sistemas, tornando possível a construção de múltiplos indicadores de saúde e análises cada vez mais complexas e apuradas do processo de saúde e doença da população.

Dentre os diversos subsistemas de saúde contidos no DATASUS, para apresentação, selecionamos seis que são de especial interesse para a vigilância em saúde.

\section{Sistema de informação de agravos de notificação}

É o mais importante para a vigilância epidemiológica, pois consolida o registro das doenças de notificação compulsória do Brasil. Esse sistema tem como objetivo padronizar a coleta e o processamento dos dados sobre doenças e agravos de notificação compulsória (DNC) em todo território nacional. É alimentado por dois instrumentos de coleta de dados, a Ficha Individual de Notificação e a Ficha Individual de Investigação. Além das DNC, o sistema é flexível e permite que seja facultado a estados e municípios incluírem outros problemas de saúde importantes em sua região.

A partir da alimentação do banco de dados do Sistema de informação de agravos de notificação (Sinan), pode-se calcular um conjunto de indicadores do estado de saúde da população (por exemplo, incidência, prevalência, letalidade e mortalidade, inclusive de doenças imunopreveníveis), bem como realizar análises, de acordo com as características de pessoa, tempo e lugar, particularmente no que tange às doenças transmissíveis de notificação obrigatória.

Veja um exemplo: a análise de casos novos de sarampo obtidos através do Sinan vai permitir gerar informações sobre as características demográficas e sociais desses casos registrados e, com isso, traçar um perfil 
da população acometida, identificar um surto e propor medidas de intervenção, como a intensificação da vacinação na região onde essas pessoas residem.

\section{Sistema de informação de mortalidade}

O Sistema de informação de mortalidade (SIM) é um sistema que tem como objetivo captar os dados de óbitos e fornecer informações sobre mortalidade. Utiliza um instrumento padronizado de coleta de dados, a Declaração de Óbito (DO).

Na DO, são coletados dados que irão gerar informações sobre as causas dos óbitos e as características demográficas e sociais dos óbitos registrados, bem como traçar um perfil de mortalidade de determinada população e, a partir disso, gerar intervenções para melhorar a condição de saúde daquela população. Por exemplo, se uma doença imunoprevenível começa a aparecer com intensidade como causa de óbito, podem ser propostas alterações nas campanhas de vacinação.

Deve-se destacar a importância do SIM nos óbitos notificados suspeitos de serem decorrentes da aplicação de um produto imunobiológico. Nessa situação, há necessidade de investigação epidemiológica minuciosa do óbito para pesquisa de estados mórbidos antecedentes e descarte de possível associação causal com o imunobiológico aplicado.

\section{Sistema de informações sobre nascidos vivos}

O número de nascidos vivos, registrado no Sistema de informações sobre nascidos vivos (Sinasc), constitui relevante informação para o campo da saúde pública. As informações sobre nascidos vivos permitem identificar problemas, avaliar riscos e orientar o planejamento das ações voltadas para a saúde materno-infantil por meio de construção e acompanhamento de indicadores de saúde, como, por exemplo, os coeficientes de mortalidade infantil e materna.

O Sinasc tem como instrumento padronizado de coleta de dados a Declaração de Nascido Vivo (DNV), cuja emissão, a exemplo da DO, é de competência exclusiva do Ministério da Saúde. Além de possibilitar o conhecimento do número de nascidos vivos, a DNV promove a coleta de dados como:

蒌 data e hora do nascimento;

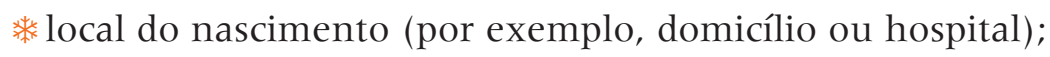

*aracterísticas do recém-nascido (por exemplo, peso, índice de Apgar);
O preenchimento da Declaração de Óbito (DO) deve ser realizado exclusivamente por médicos, exceto em locais onde não existam esses profissionais, situações nas quais poderá ser preenchida por oficiais de cartórios de registro civil, sendo também assinada por duas testemunhas. A obrigatoriedade de preenchimento desse instrumento é determinada pela Lei Federal n. 6.015/73. 
A Lei n. 9534, de 1997, garante o direito à primeira certidão de nascimento gratuita para todos os cidadãos brasileiros.
A ocorrência de reações não desejadas desencadeadas pela vacinação (EAPV) será discutida no Capítulo 3, "Vigilância epidemiológica em eventos adversos pós-vacinação" do livro Rede de Frio: gestão, especificidades e atividades (SILVA; FLAUZINO, 2016).
䊁 dados sobre a gestação (por exemplo, número de consultas prénatal, duração da gravidez);

燐 características da mãe (por exemplo, idade).

Tanto a emissão da DNV como o seu registro em cartório são realizados no município de ocorrência do nascimento. Deve ser preenchida pelos hospitais e por outras instituições de saúde que realizam o parto e nos Cartórios de Registro Civil, quando o nascimento da criança ocorre no domicílio.

\section{Sistema de informações hospitalares}

O Sistema de informações hospitalares (SIH/SUS) não foi concebido sob a lógica epidemiológica, mas com o propósito de operar o sistema de pagamento de internação nos hospitais contratados pelo Ministério da Previdência. Posteriormente, foi estendido aos hospitais filantrópicos, universitários e de ensino, e aos hospitais públicos municipais, estaduais e federais. É um sistema de registro de internações hospitalares ocorridas no sistema de saúde público do país.

Esse sistema, apesar de apresentar essa restrição, é de extrema importância para o conhecimento do perfil dos atendimentos da rede hospitalar. Seus registros representam cerca de $70 \%$ a $80 \%$ das internações hospitalares de todo o Brasil e, por esse motivo, possibilitam a avaliação da assistência hospitalar e da vigilância epidemiológica.

Por meio de seu documento principal, a Autorização de Internação Hospitalar, são coletadas, dentre outros dados, as causas de internação do paciente, possibilitando que, a partir da análise de tais dados, obtenha-se um perfil de morbidades que levaram à hospitalização em determinada população.

Para a rede de frio, tem-se mostrado útil, por registrar internações devidas a eventos adversos pós-vacinais (EAPV), permitindo maior conhecimento sobre a vacina e o desenvolvimento de pesquisas que ajudem na identificação dos eventos decorrentes dos imunobiológicos aplicados.

\section{Sistema de informações ambulatoriais do SUS}

O Sistema de informações ambulatoriais do SUS (SIA/SUS) é um sistema de registro dos atendimentos e procedimentos ambulatoriais realizados pelos prestadores públicos e privados, contratados e conveniados pelos SUS. Foi implantado dentro de uma lógica contábil para o controle de gastos com a assistência ambulatorial. 
Esse sistema registra o procedimento ambulatorial realizado (desagregado em atos profissionais), por exemplo, número de consultas médicas por habitante ao ano, número de consultas médicas por consultório, número de exames/terapias realizados pelo quantitativo de consultas médicas.

Nesse sistema, estão incluídos os procedimentos de aplicação de imunobiológicos por unidade de saúde, contudo as informações produzidas referem-se unicamente às unidades de saúde do SUS, e não são registrados e armazenados dados individuais de atendimento.

O SIA/SUS possibilita a organização e o planejamento dos gestores locais para a avaliação de procedimentos ambulatoriais, além da análise qualitativa e quantitativa das ações de saúde, ou seja, é especialmente útil para análise de dados operacionais para controle e avaliação do SUS.

\section{Sistema de informações do Programa Nacional de Imunizações}

Fornece dados relativos à cobertura vacinal de rotina e em campanhas, taxa de abandono e controle do envio de boletins de imunização. Dispõe de um subsistema que permite controlar o estoque e a distribuição de imunobiológicos para fins gerenciais.

\section{Considerações finais}

O principal objetivo deste capítulo foi proporcionar a você uma visão introdutória sobre como são obtidos dados e como são geradas informações que balizam, direta ou indiretamente, diretrizes e políticas públicas que buscam melhorar a saúde da população.

Desse modo, analisamos os indicadores, vimos para que se destinam, suas finalidades, apresentando os mais utilizados na área da saúde. Observamos que existem outros tipos de indicadores que não são dessa área, mas que são fundamentais para entendermos e analisarmos o processo de saúde e doença da população.

Demonstramos, também, os principais sistemas de informação em saúde, como foram concebidos, seus propósitos e instrumentos de coleta. Enfatizamos a utilidade dos sistemas para a formulação e avaliação de políticas, planos e programas de saúde, para subsidiar o processo de tomada de decisões.

Agora você, profissional de saúde da rede de frio, terá mais ferramentas para compreender por que determinadas situações relativas à saúde da população envolvem a dinâmica de seu processo de trabalho. Entendendo
O sistema de informações do Programa Nacional de Imunizações é discutido com maior profundidade no Capítulo 5, "Sistemas de Informações do Programa Nacional de Imunizações", do livro Rede de Frio: gestão, especificidades e atividades (SILVA; FLAUZINO, 2016). 
que os indicadores epidemiológicos, obtidos através dos sistemas de informação em saúde, são ferramentas poderosas, práticas e úteis a serviço do SUS, devemos nos apropriar deles para enriquecer o desenvolvimento de nossas ações. Isso é importante porque, além de estratégica, nossa atuação deve ter, em sua essência, responsabilidade, maturidade e respeito com a saúde da nossa população.

\section{Referências}

ALMEIDA FILHO, N.; ROUQUAYROL, M. Z. Introdução à epidemiologia. 4. ed. rev. e ampl. Rio de Janeiro: Guanabara Koogan, 2006.

BEAGLEHOLE, R. et al. Epidemiologia básica. Washington, D.C.: Organização Mundial da Saúde, 1994.

BRASIL. Ministério da Saúde. Secretaria de Vigilância em Saúde. Guia de vigilância epidemiológica. 7. ed. Brasília, DF, 2009.

BRASIL. Ministério da Saúde. Secretaria de Atenção à Saúde. Departamento de Atenção Básica. Vigilância em saúde: zoonoses. Brasília, DF, 2009.

BRASIL. Ministério da Saúde. Secretaria de Vigilância em Saúde. Departamento de Análise de Situação de Saúde. Saúde Brasil 2009: uma análise da situação de saúde e da agenda nacional e internacional de prioridades em saúde. Brasília, DF, 2010.

CID 10: Classificação estatística internacional de doenças e problemas relacionados à saúde. Brasília, DF: Organização Mundial da Saúde, 2008.

COMISSÃO NACIONAL DE DETERMINANTES SOCIAIS DA SAÚDE (Brasil). Secretaria Técnica. Iniquidades em Saúde no Brasil: nossa mais grave doença. Rio de Janeiro, 2006. Texto produzido para o lançamento da Comissão, ocorrido em 15/3/2006. Disponível em: <http://www. determinantes.fiocruz.br/acoes.asp >. Acesso em: 15 set. 2015.

DATASUS. O Datasus. Brasília, DF, 2011. Disponível em: <http://www2.datasus.gov.br/DATASUS/ index.php?area=01>. Acesso em: 25 jul. 2016.

DATASUS. Histórico/apresentação. Brasília, DF, [201-]. Disponível em: <http://datasus.saude.gov.br/ datasus>. Acesso em 25 jul. 2016.

GORDIS, L. Epidemiologia. Rio de Janeiro: Revinter, 2010.

MEDRONHO, R. A. et al. Epidemiologia. 2. ed. São Paulo: Atheneu, 2009.

ORGANIZAÇÃO PAN-AMERICANA DA SAÚDE. Módulos de princípios de epidemiologia para o controle de enfermidades: módulo 2: saúde e doença na população. Brasília, DF, 2010.

REDE INTERAGENCIAL DE INFORMAÇÕES PARA A SAÚDE (Brasil). Objetivos. [S.I.], 2012. 25 jul. 2016. 
REDE INTERAGENCIAL DE INFORMAÇÕES PARA A SAÚDE (Brasil). Indicadores básicos para a saúde no Brasil: conceitos e aplicações. 2. ed. Brasília, DF: Organização Pan-Americana da Saúde, 2008.

ROUQUAYROL, M. Z.; ALMEIDA FILHO, N. Epidemiologia \& saúde. 6. ed. Rio de Janeiro: MEDSI, 2003.

ROUQUAYROL, M. Z.; SILVA, M. G. C. Epidemiologia \& saúde. 7. ed. Rio de Janeiro: Medbook, 2013.

SILVA, M. N.; FLAUZINO, R. F. (Org.). Rede de frio: gestão, especificidades e atividades. Rio de Janeiro: Ed. Fiocruz, 2016. No prelo.

SANTOS, A. M. A. Causalidade entre renda e saúde: uma análise através da abordagem de dados em painel com os Estados e Municípios Brasileiros. 2010. Dissertação (Mestrado em Economia) - Universidade Federal de Alagoas, Faculdade de Economia, Administração e Contabilidade, Programa de Pós-Graduação em Economia, Maceió, 2010.

SANTOS, P. A. L. B. Epidemiologia aplicada nos serviços de saúde. São Paulo: Martinari, 2012. 